\title{
The Topological Consistence of Path Connectedness in Regular and Irregular Structures
}

\author{
Helmut Kofler \\ 1050 Vienna, Grohg. 3/7, Austria \\ kof@vienna.at
}

\begin{abstract}
In this paper an overview of regular adjacency structures compatible with topologies in 2 dimensions is given. This means that the different discrete structures are investigated on the equivalence of topological-connectedness and path-connectedness which is induced by the underlying adjacency.

In the second part a method is suggested how to construct topologies on irregular graphs and their compatibility to the adjacency structure is analyzed. Examples of basic adjacency structures are given. These topologies on regular grids and especially on irregular graphs are appropriate to be applied to image hierarchies in the field of image processing.
\end{abstract}

\section{Introduction}

Is there a topological structure which fits best on a discrete structure? There is no homogeneous topology on a grid like $\mathbb{Z}^{2}$, except the discrete topology, because of the result: any local finite $T_{1}$-topology is discrete. But there exist

- homogeneous topological structures like the semi-topological space [8] and

o non-homogeneous $\left(T_{0}\right.$ minus $\left.T_{1}\right)$-topologies like cellular models $[2,5,6]$, the star-topology[1] and the $n$ dimensional Marcus-Wyse topology[12].

Cellular models can be translated into adjacency graphs with an easy construction which is demonstrated in Fig. 1.

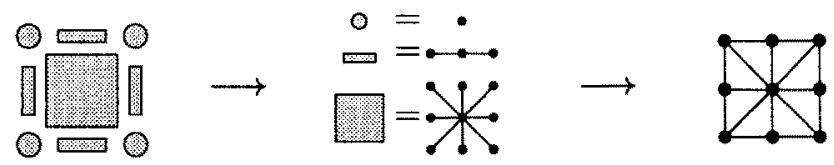

Fig. 1. Cellular models and adjacency neighborhood structures.

In the 4-grid $\left(x_{1}, x_{2}\right) \in \mathbb{Z}^{2}$ the 2 dimensional Marcus-Wyse topology $\tau_{M W}$ is induced by the sets $U$ :

$$
U \epsilon \tau_{M W} \equiv\left\{\begin{aligned}
U_{4}\left(\left(x_{1}, x_{2}\right)\right) & : \text { if } x_{1}+x_{2} \text { is even } \\
\left\{\left(x_{1}, x_{2}\right)\right\} & : \text { else }
\end{aligned}\right.
$$

where $U_{4}\left(\left(x_{1}, x_{2}\right)\right):=\left\{\left(x_{1}, x_{2}\right),\left(x_{1}-1, x_{2}\right),\left(x_{1}, x_{2}+1\right)\left(x_{1}+1, x_{2}\right),\left(x_{1}, x_{2}-1\right)\right\}$, $\left(x_{1}, x_{2}\right) \in \mathbb{Z}^{2}$ (even and odd can be exchanged). The topology $\tau_{M W}$ is not homogeneous, but the property that path-connectedness is equivalent to topological connectedness is given in this environment. This is not true for 6 or 8 -adjacency [8, 
10,12]. The equivalence of path-connectedness and topological connectedness of regular graphs will be summarized in theorem 1 in section 3 . In section 4 a topology on an irregular graph is constructed and the discussion about equivalence of path-connectedness and topological connectedness will be formulated in theorem 2. Digital image-operations and algorithms like image hierarchies are intended to be applied on such digital topological structures.

\section{Basic definitions}

In order to investigate regular but also irregular adjacencies a graph structure $G(V, E)$ is used where $V(G)$ is the set of vertices and $E(G)$ is the set of edges. Instead of the geometical information the adjacency neighborhood relation beconues important (Fig. 2).
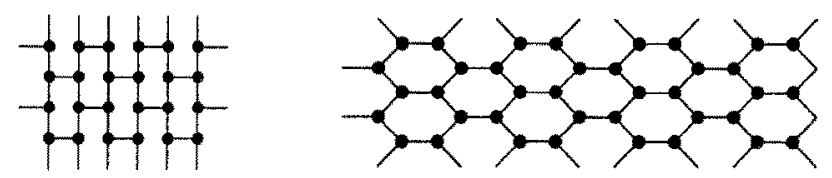

Fig. 2. Two "topological equivalent" 3-adjacency"pattern.

On a graph $G(V, E)$ neighborhoods can be formulated in a natural way: A neighborhood of a vertex $U(v), v \epsilon V$ is defined as a set of vertices $w \epsilon V$ with $w$ is adjacent to $v$, i.e. $U(v):=\{w \epsilon V(G) ;(w, v) \epsilon E\} \cup\{v\}$.

The vertex $v$ is per definitionem element of the neighborhood itself. The degree of vertex $\operatorname{deg}(v), v \in V$ is the number of all adjacent vertices $w$. In a regular structure the degree of every vertex $v$ is constant:

$$
\operatorname{deg}(G):=\min \{\operatorname{deg}(v) ; v \epsilon V\}=\max \{\operatorname{deg}(v) ; v \epsilon V\} .
$$

If the $\operatorname{deg}(v) \geq 1, v \in V$, such a neighborhood $U(v)$ is called a star set.

Definition of path-connected on the graph $G(V, E)$

Let $W$ be a subset of $V(G)$. Suppose that $v, w \epsilon W$. Then $W$ is path-connected, if to each $v, w \in W$ there exists a path in $W$ that combines $v$ and $w$. A path in $W$ is a finite sequence of vertices $v=v_{1}, v_{2}, \cdots, v_{n}=w$ that $v_{i} \in W$ is a neighbor of $v_{i-1}, 1<i \leq n$.

The graph $G(V, E)$ regarded as a neighborhood structure where the basic neighborhoods are defined by the sets of all adjacencies of a vertex $v$ ( $v$ is included) gives a topological structure. Because of the loss of the intersection property the neighborhood structure cannot be a topology. But there exist topologies on graphs like the Marcus-Wyse topology $\tau_{M W}$ on a 4-grid. A n-grid means an infinite plane graph $G_{n}\left(\mathbb{Z}^{2}, E\right)$ in the plane with $\operatorname{deg}\left(G_{n}\right)=n$.

Definition of topological-connected on the graph $G(V, E)$

Let $\tau$ be a topology on the graph $G(V, E)$. A subset $W$ of $V$ is connected if there are no two disjoint (open) sets $O_{1}, O_{2} \in \tau$ existing that $\left(O_{1} \cap W\right) \cup\left(O_{2} \cap W\right)=W$.

In a graph $G(V, E)$ a topological-connected set should also be path-connected. We call the topology $\tau$ on the graph $G$ compatible to the underlying adjacency 
structure (defined by $E$ ) if path-connectedness is equivalent to topological connectedness $[4,11]$.

\section{Regular Adjacency neighborhood structures in 2 dimensions}

In the following theorem the connectedness results for n-adjacency neighborhood structures in 2 dimensions are summarized.

\section{Theorem 1 - connectedness on regular graphs in 2 dimensions}

Let $G(V, E)$ be a regular infinite graph in the plane with degree $\operatorname{deg}(G)=n$. There exists exactly one type of topology $\tau$ for each case of adjacency $n$ smaller or equal 4 that the topology $\tau$ is compatible to the underlying adjacency neighborhood structure, i.e. path-connectedness and topological-connectedness is equivalent. (One type means homeomorphic spaces, e.g. regarding the different MarcusWyse topologies resp. odd and even.)

Especially for the case $n=4$ the proof is given in detail in the literature in $[4$, $8,10,12]$. For the cases $n \geq 0$ the same strategy is possible. After the proof we will give a list of these topologies.

\section{Structure of the proof:}

Let $v \epsilon V(G)$. It is to show, if the set $U(v)$ is the smallest neighborhood of $v$, which is an open set in a topology $\tau$ compatible with n-adjacency, then $U(v)$ must be smaller than the star set of $v$, all the adjacent vertices of $v$ and $v$ included itself: $\quad U(v) \subseteq\{w \epsilon V(G) ; w=v$ or $(w, v) \epsilon E\}$.

Let $V_{1}:=\{v\}$ and $V_{2}:=V-\{w \epsilon V(G) ; w=v$ or $(w, v) \epsilon E\}$, then both $V_{1}$ and $V_{2}$ are connected but $V_{1} \cup V_{2}$ is not. It follows that there is a topological neighborhood of $v$ in any topology compatible with $n$-adjacency which is disjoint with $V_{2}$. This means that such a set $U(v)$ must be a subset of $\{w \in V(G) ;(w, v) \epsilon E\} \cup\{v\}$.

In a further step it is to show either $U(v)=\{v\}$ or $U(v)=\{w \epsilon V ;(w, v) \epsilon E\} \cup$ $\{v\}:$ If $U(v) \neq\{v\}$ and $U(v) \neq\{w \epsilon V ;(w, v) \in E\}$ there exist $a w^{\prime} \in V$ with $\left(w^{\prime}, v\right) \epsilon E$ but $w^{\prime} \not U(v)$. This means $v$ and $w^{\prime}$ can be separated by $U(v) \epsilon \tau$ and a set $U\left(v^{\prime}\right) \epsilon \tau, v^{\prime} \in V$ with $\left(v^{\prime}, w^{\prime}\right) \epsilon E$ - which is contradicted to $v$ and $w^{\prime}$ are $n$-adjacent. This set $U\left(v^{\prime}\right)$ with $v^{\prime} \notin U(v)$ exists, because we can assume that $\operatorname{deg}\left(w^{\prime}\right) \geq 2$ and the structure $G$ is infinite.

It is obvious that the singleton sets $\{v\}, v \in V$ cannot constitute the neighborhoods for all $v \in V$, we would obtain the discrete topology. The discrete topology is only compatible to 0 and 1 adjacency. It is also obvious that the star sets $U(v)=\{w \epsilon V(G) ;(w, v) \epsilon E\} \cup\{v\}, v \epsilon V$ cannot be the smallest neighborhoods because of the intersection property of a topology. Thus, in every topology compatible with n-adjacency (except the case $n=0$ of course) we must have both the singleton smallest neighborhoods and the "star-like" neighborhoods.

How to take the neighborhoods for the case $n \geq 2$ we guide along the well known case of 4-adjacency and we show an example e.g. for the case $n=5$ (Fig. 3). Below we will give a list of all adjacencies.

At last for $n \leq 4$ it would be necessary to proof that the constructed topology is compatible with the underlying $\mathrm{n}$-adjacency. This has been done in detail for $n=4 \mathrm{in}[12]$. 
In the case of higher adjacencies $n \geq 5$ a counter-example fulfills the proof. A counterexample for the case $n=5$ is shown in Fig. 5 .
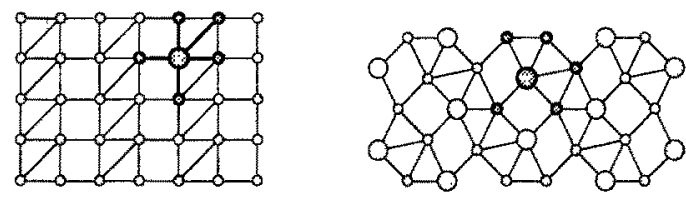

Fig. 3. Two versions of a 5-adjacency pattern, "star-sets" are figured out.

Instead of looking for counter-examples for higher adjacencies we can argue that if two graphs $G_{1}$ and $G_{2}$ give two adjacency structures and $G_{2}$ is finer than $G_{1}$, then the absence of a compatible topology for $G_{1}$ implies the absence of a compatible topology for the finer structure $G_{2}$.

The different n-adjacency structures (regular graphs with degree n):

- Adjacency, i.e. 0-Adjacency Let $V \subseteq \mathbb{Z}^{2}$. Each vertex $v \in V$ is related only to itself. There is exactly one topology on $V$ the discrete topology which fulfills path-connectedness is equivalent to topological-connectedness.

- Adjacency, i.e. 1-Adjacency There exists a structure like the discrete topology which fulfills path-connected is topological-connected. Each pair of adjacent vertices $\{v, w\}$ is in that topology. To arrange that the topology fulfills the $T_{o}$ separation axiom we choose one single point set $\{v\}$ out of it. Sets of vertices of different pairs are disconnected in the sense of the adjacency and the topology - this means every set with 3 vertices is disconnected.

$\longrightarrow$ Adjacency, i.e. 2-Adjacency There are topological structures in 2 dimensions of the type of paths which inherit the 1-dimensional Marcus-Wyse topology - path-connectedness is equivalent to topological-connectedness. The case of finite paths if they are closed is discussed below.
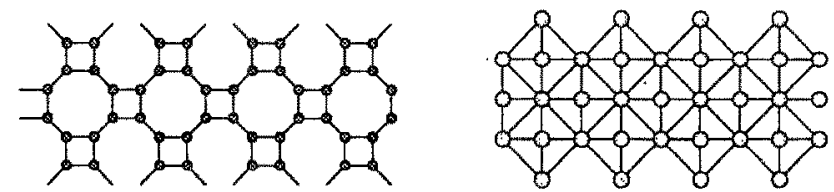

Fig. 4. A 3-adjacency-pattern which is topologically (but not geometrically) regular and its (irregular) dual graph, a 4 combined with an 8 adjacency pattern, the vertices are the regions of the graph.

Regarding a 3-adjacency pattern

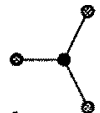

Adjacency, i.e. 3-Adjacency

(Fig. 4), a regular infinite graph with degree 3 , there exists one topology on the graph which is compatible to the underlying adjacency structure.

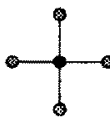

Adjacency, i.e. 4-Adjacency

In a 4-adjacency pattern (a 4-grid) the 2-dimensional Marcus-Wyse topology is the only topology which fulfills 
path-connected is equivalent to topological-connected. This result compared with the 8-adjacency is a often discussed case in literature, thinking of Jordan's curve theorem.
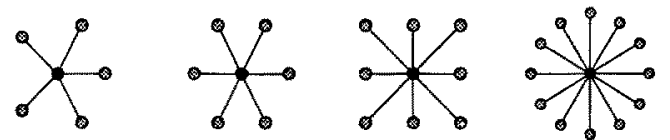

\section{Adjacencies, i.e. 5, 6, 8, 12-Adjacency}

In n-adjacency structures $n \geq 5$ path-connectedness is not equivalent to the topological connectedness. Often discussed adjacencies are 6,8 and 12 adjacencies.

A counter-example for a 5-adjacency structure $G_{5}(V, E)$ (Fig. 3) is shown in Fig. 5, four star sets are chosen $U(i)=\{w \epsilon V ;(w, i) \epsilon E\} \cup\{i\}, 1 \leq i \leq 4$, $i \epsilon V\left(G_{5}\right)$. The set of the 2 points (the intersection of $U(2)$ and $\left.U(4)\right)$ is 5connected resp. the adjacency relation,

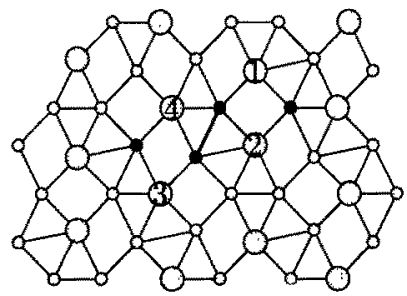

Fig. 5. A counter-example for the case $n=5$.

but can be separated by the sets $U(1) \cap U(2) \epsilon \tau$ and $U(3) \cap U(4) \epsilon \tau$ (The intersection points are marked in black). Which means that the set of these 2 points is not connected in a topological sense.

\section{Regular Adjacency neighborhood structures in higher dimensions}

In higher dimensions obviously the $n$ dimensional Marcus-Wyse topology is compatible to the underlying $2 n$ adjacency structure. But in $n$ dimensions there exist compatible topologies on higher adjacencies $\geq 2 n$, which can be seen in the case $n=3$ adding one edge in the direction of a space-diagonal that each vertex has degree 7 .

On adjacency structures where intersections of star sets are sets of single points or at least points which are not adjacent there exists a compatible topology. (This will be seen in the next section where a topology will be constructed.)

But on the other side in every dimension there must be a highest number $N \in \mathbb{N}$ where a compatibility of a topology and a $\mathrm{N}$-adjacency can be shown. If the degree $n$ is too high there will appear substructures excluded in the 2 dimensional case.

\section{A topology on irregular adjacencies in 2 dimensions}

Let $G(V, E)$ be an irregular (plane) graph. The graph can be finite or infinite (like the dual graph in Fig.4). A construction for a topology will be given on an irregular graph in the plane and the connectedness condition will be discussed. The relative topology on an arbitrary subset $W \subseteq V$ of the graph $G$ resp. the 
topology $\tau$ gives topologies on small sets, also the star sets. On the other hand the topology on the whole graph $G$ can be composed in a suitable way by the help of these "basic" topologies on the small sub-graphs.

First we give some examples of such topologies $\tau$ on small graphs (not all unions of sets in $\tau$ are mentioned - only basic sets, the whole set itself and the empty set $\emptyset$ ):

$$
\begin{aligned}
& \text { (1)-(2) } \tau_{1}=\{\{1,2\}, \emptyset,\{2\}\}, \\
& \text { (3) (2) } \tau_{1}=\{\{1,2,3\}, \emptyset,\{2,3\},\{3\}\} \text {, } \\
& \text { 1) } \tau_{1}=\{\{1,2,3\}, \emptyset,\{2\},\{3\}\}, \\
& \text { (3) } \tau_{2}=\{\{1,2,3\}, \emptyset,\{2,1\},\{3,1\},\{1\}\} \text {, } \\
& \text { (5) } \tau_{1}=\{\{1,2,3,4,5\}, \emptyset,\{2\},\{3\},\{4\},\{5\}\} \text {, } \\
& \tau_{2}=\{\{1,2,3,4,5\}, \emptyset,\{2,1\},\{3,1\},\{4,1\},\{5,1\},\{1\}\}, \\
& \text { (2) (3) } \begin{array}{l}
\tau_{1}=\{\{1,2,3,4,5\}, \emptyset,\{2\},\{2,3,4\},\{4\},\{4,5\}\}, \\
\tau_{2}=\{\{1,2,3,4,5\}, \emptyset,\{2,3,4,5\},\{3,4,5\},\{4,5\},\{5\}\} .
\end{array}
\end{aligned}
$$

These topologies $\tau_{1}$ are constructed by typical star sets with its center assumed by $1 \epsilon V$. The topology $\tau_{1}$ of the first, third and fourth set are the MarcusWyse topology. The topology $\tau_{1}$ on the last set uses the 1 dimensional MarcusWyse topology: Comparing the topology $\tau_{1}$ with $\tau_{2}, \tau_{2}$ is not compatible to the underlying adjacency structure, because e.g. vertex 2 and 4 cannot be separated by set $U, U^{\prime} \epsilon \tau_{2}$.

\section{Topological connectedness on closed paths}

There exist two types of closed paths $G_{c y c l e}(V, E)$, these with odd vertices and even vertices. An example shows the way how to treat getting compatible topologies in the even case:<smiles>[CH]1COC1</smiles>

$$
\tau=\{\{1,2,3,4\}, \emptyset,\{1,2,4\}\{2\},\{2,3,4\},\{4\}\} .
$$

The 1 dimensional Marcus-Wyse topology again gives the solution. The centers of the star sets are $1,3 \epsilon \mathrm{V}$. But in the odd case the construction of a compatible topology is more ambitious:

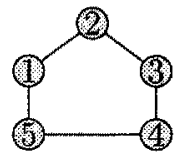

$$
\tau=\{\{1,2,3,4,5\}, \emptyset,\{2,1,5,4\},\{2\},\{2,3,4,5\},\{4,5\},\{5\}\} .
$$

The centers of the star sets are 1,3,4€V. Larger closed paths (odd or even) can be reached by exchanging vertex 2 with an arbitrary set of odd numbers of vertices equipped with the 1 dimensional Marcus-Wyse topology. 
In the following theorem the construction of a topology is given and it is demonstrated if the topology is compatible to its graph structure.

\section{Theorem 2 - connectedness on irregular planar graphs}

Let $G(V, E)$ be a general planar graph. There exist topologies $\tau$ on the graph $G$, where the compatibility of path-connectedness and topological-connectedness is maintained if the graph exists of vertices $v \epsilon V$ of degree smaller or equal 4. In general a topology $\tau$ on $G(V, E)$ cannot be unique.

\section{Proof}

Let $G(V, E)$ be an irregular plane graph which is finite. The construction of the topology $\tau$ :

- First $n=1$ we choose one vertex $v_{1} \epsilon V$. Then we build the star set

$$
U\left(v_{1}\right)=\left\{w \in V(G) ;\left(w, v_{1}\right) \epsilon E\right\} \cup\left\{v_{1}\right\} \in \tau .
$$

- In a second step $n=2$ we choose a vertex $v_{2} \epsilon V$ with $v_{2} \notin U\left(v_{1}\right)$ but adjacent to a vertex $w \epsilon U\left(v_{1}\right)$ and we build the star set $U\left(v_{2}\right) \in \tau$.

The intersection of $U\left(v_{1}\right)$ and $U\left(v_{2}\right)$ is a subgraph where we install a topology as mentioned above. Is the intersection a set of one vertex $v$ then $\{v\}$ is in the topology $\tau$. On an intersection of adjacent vertices $v, w$ the above simple topology will be installed. In the next steps we take vertices $v \epsilon V$ adjacent to a vertex $\left.w \epsilon U\left(v_{1}\right)\right)$, if there are some left.

- In $n=3$ we take a vertex $v_{3} \epsilon V$ with $v_{3} \notin U\left(v_{1}\right) \cup U\left(v_{2}\right)$ and $v_{3}$ is adjacent to a vertex $w \epsilon U\left(v_{1}\right) \cup U\left(v_{2}\right)$.

- In the $n$ step we choose a vertex $v_{n} \epsilon V$ with the condition $v_{n} \not\left(U\left(v_{1}\right) \cup \cdots \cup\right.$ $\left.U\left(v_{n-1}\right)\right)$ and $v_{n}$ is adjacent to a vertex $w \epsilon\left(U\left(v_{1}\right) \cup \cdots \cup U\left(v_{n-1}\right)\right)$.

- We finish with finite $N \geq 0$ steps since $G(V, E)$ is finite. If an infinite graph - like the infinite graph in Fig. 4 - is given, a construction condition of $G$ to the infinity must be used.

The topology $\tau$ cannot be unique. The topology depends on the starting point as well as the choice of the vertices $v_{n}$ in every step. Remember at this place the Marcus-Wyse topology which is not unique too. There are 2 topologies on $\mathbb{Z}$ (starting with odd or even). In the present case of irregular graphs $G$, when it is a finite structure, of course, there also must be a finite number of topologies. The condition considering only vertices $v \epsilon V(G)$ with $\operatorname{deg}(v) \leq 4$ is sufficient to exclude all bad cases which can appear disturbing a compatibility to the constructed $\tau$, e.g. the case of the counter-example in Fig. 5. But it is not a necessary condition which can be seen easily in the following Fig.:

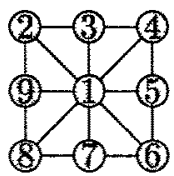

$$
\tau=\{\{1,2,3, \cdots, 8,9\}, \emptyset,\{2,3,4\},\{4,5,6\},\{6,7,8\},\{8,9,2\}\}
$$

On the graph vertex 1 has $\operatorname{deg}(1)=8$, but $\tau$ is compatible to it's adjacency structure. A transformation of higher adjacencies into less or equal 4 can be proposed in the following.

Transformation of higher adjacencies into $\leq 4$ adjacencies

Let $G(V, E)$ be a graph in the plane then it is possible to transform locally large 
adjacencies into adjacencies less or equal 4. This will be demonstrated in the following drawing:
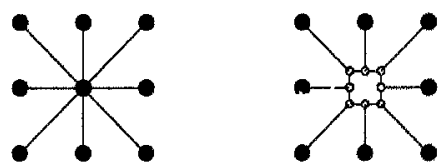

Fig. 6. A transformation of a vertex with degree 8 .

A vertex $v \in V$ with degree $\operatorname{deg}(v)=n$ will be extended into a subgraph $G_{v}$ with $n$ vertices and $\operatorname{deg}\left(G_{v}\right)=3$. The vertex $v$ merged into the subgraph $G_{v}$ can be interpreted as a view on the unit in detail - more than one input and output interfaces are seen. The edges can be established with infinitesimal length to emphasize the character of the unit.

\section{Conclusion}

The equivalence of path-connectedness and topological-connectedness is discussed in regular adjacency structures in 2 dimensions. Result must be given for higher dimensions in future. A topology is given to irregular graphs with a condition that path-connectedness and topological-connectedness must be equivalent.

Digital image-operations and algorithms like image hierarchies can be applied on such irregular topological structures. Especially in the dual graph contraction [7] an implementation of the algorithm, which builds topologies on irregular graphs, is aimed.

\section{References}

1. E. Ahronovitz, J-P. Aubert, Chr. Fiorio. The Star-Topology: a topology for image analysis. 5th International Workshop, DGCI'95, 1995.

2. P. Alexandroff, H. Hopf. Topologie, Erster Band. Springer, Berlin, 1935.

3. E. Cech. Topological Spaces. Interscience, Wiley, NewYork, 1966.

4. J.M. Chassery. Connectivity and consecutivity in digital picture. Computer Graphics and Image Processing 9, 1979.

5. E. Khalimsky, R. Kopperman, P.R. Meyer. Computer graphics and connected topologies on finite ordered sets. Topology Appl. 36, 117, 1980.

6. V.A. Kovalevsky. Finite topology as applied to image analysis. Computer Vision, Graphics and Image Processing 46, 1989.

7. W. Kropatsch. Building Irregular Pyramids by Dual Graph Contraction. IEE Proceedings Vis. Image Signal Process., Vol. 142, No. 6, 1995.

8. L. Latecki. Digitale und Allgemeine Topologie in der bildhaften Wissensrepräsentation. Ph.D.-Thesis, Hamburg, 1992.

9. L. Latecki. Topological connectedness and 8-connectedness in digital pictures. Computer Vision, Graphics and Image Processing: Image Understanding 57, 1993.

10. P. Ptak, H. Kofler, W. Kropatsch. Digital Topologies Revisited. 7th International Workshop, DGCI'97, Montpellier, France, 1997.

11. A. Rosenfeld. Digital topology. Am. Math. Monthly 86, 1979.

12. F. Wyse, D. Marcus et al. Solution to Problem 5712. Am. Math. Monthly 77, 1970. 